\title{
Characteristic creep behavior of nanocrystalline metals found for high-density gold
}

\author{
S. Sakai, ${ }^{1, *}$ H. Tanimoto, ${ }^{1,3}$ E. Kita, ${ }^{2,3}$ and H. Mizubayashi ${ }^{1, \dagger}$ \\ ${ }^{1}$ Institute of Materials Science, University of Tsukuba, Tsukuba, Ibaraki 305-8573, Japan \\ ${ }^{2}$ Institute of Applied Physics, University of Tsukuba, Tsukuba, Ibaraki 305-8573, Japan \\ ${ }^{3}$ Center for Tsukuba Advanced Research Alliance, University of Tsukuba, Tsukuba, Ibaraki 305-8573, Japan
}

(Received 27 June 2002; revised manuscript received 7 October 2002; published 18 December 2002)

\begin{abstract}
Nanocrystalline $(n)$ Au specimens with a density of $19.4 \pm 0.2 \mathrm{~g} / \mathrm{cm}^{3}$ and a mean grain size of about $20 \mathrm{~nm}$ were prepared below $300 \mathrm{~K}$ by the gas deposition method, where two types of $n$-Au specimens were obtained as a function of a deposition rate, the type- $H$ specimens above $800 \mathrm{~nm} / \mathrm{s}$ and the type- $L$ specimens below 800 $\mathrm{nm} / \mathrm{s}$. The anelastic and the plastic creep responses are similar qualitatively but different quantitatively between the type- $H$ and type- $L$ specimens. The anelastic strain $\varepsilon_{a n, \mathrm{~GB}}$, associated with the grain boundary (GB) regions, increases linearly with $\left(T-T_{a n 1}\right)\left(\sigma_{a p}-\sigma_{a n 1}\right)$, when the temperature $T$ is higher than a threshold temperature $T_{a n 1}$ of $200 \mathrm{~K}$ and the applied stress $\sigma_{a p}$ is higher than a threshold stress, $\sigma_{a n 1}$, of a few MPa. The ratio of $\varepsilon_{a n, \mathrm{~GB}}$ to the elastic strain is as large as 1.1 for the type- $H$ specimens and 0.2 for the type- $L$ specimens at $320 \mathrm{~K}$ for $\sigma_{a p} \gg \sigma_{a n 1}$. The activation energy for the GB anelastic process is $0.2 \mathrm{eV}$. We surmise that cooperative motions of many atoms in the GB regions are responsible for $\varepsilon_{a n, \mathrm{~GB}}$, and both $T_{a n 1}$ and $\sigma_{a n 1}$ show a distribution depending on the number of atoms associated. The plastic creep rate $\varepsilon^{\prime}$ vs $\sigma_{a p}$ data show a letter S-like curve. We classified the creep response into three categories, region I for the linear creep rate region for $\sigma_{a p}$ between $\sigma_{p c 1}$ and $\sigma_{p c 2}$, region II for the transient creep rate region for $\sigma_{a p}$ between $\sigma_{p c 2}$ and $\sigma_{p c 3}$, and region III for the saturation creep rate region for $\sigma_{a p}$ between $\sigma_{p c 3}$ and $\sigma_{y}$. The threshold stresses $\sigma_{p c 1}$ and $\sigma_{p c 2}$ and the yield stress $\sigma_{y}$ are about 30,150 , and $360 \mathrm{MPa}$ for the type- $H$ specimens, and about 60 , 300 , and $500 \mathrm{MPa}$ for the type- $L$ specimens, respectively. $\sigma_{p c 3}$ is slightly lower than $\sigma_{y}$. From scanning tunneling microscopy images, we surmise that the localized GB slip takes place in region I, and the mean separation between the localized GB slips decreases with increasing $\sigma_{a p}$ in region II and becomes comparable with the mean grain size in region III. The plastic creep in region III may be explained by the Ashby creep. The present view for the creep behavior explains the low-temperature creep behavior of fcc $n$ metals.
\end{abstract}

DOI: $10.1103 /$ PhysRevB.66.214106

PACS number(s): 62.25.+g, 62.20.Hg, 62.20.Dc, 61.46. $+\mathrm{w}$

\section{INTRODUCTION}

The volume fraction of grain-boundary (GB) regions increases with the decreasing mean grain size, and the mechanical property of nanocrystalline $(n)$ metals may be governed by the corresponding property of the GB regions. A plastic creep behavior is known as one of the grain-sizesensitive mechanical properties. The Coble creep ${ }^{1}$ or Ashby model $^{2}$ predicts that the Ashby creep rate $\varepsilon_{\text {Ashby }}^{\prime}$, for the homogeneous GB diffusion creep is given by

$$
\varepsilon_{\text {Ashby }}^{\prime}=\left(\varepsilon_{\text {Ashby }, 0}^{\prime} / k T\right) \exp \left(-E_{\mathrm{GB}} / k T\right)\left(\sigma_{a p}-\sigma_{0, \text { Ashby }}\right),
$$

where $\varepsilon_{\text {Ashby }, 0}^{\prime}=330 \Omega \delta D_{\mathrm{GB}, 0} / d^{3}$ and $\sigma_{0, \mathrm{Ashby}}=0.72 \Gamma / d$. $D_{\mathrm{GB}, 0}$ and $E_{\mathrm{GB}}$ are the pre-exponential factor and the activation energy appearing in the GB diffusion coefficient, $D_{\mathrm{GB}}$ $=D_{\mathrm{GB}, 0} \exp \left(-E_{\mathrm{GB}} / k T\right)$, where $k$ is the Boltzmann constant and $T$ is the temperature. $\Gamma$ is the mean GB energy per unit area, $\delta$ is the mean thickness of the GB regions, $\Omega$ is the atomic volume, $d$ is the mean grain size. The Ashby threshold stress $\sigma_{0, \text { Ashby }}$ is introduced under the assumption of the intermediate state for the grain switching. That is, $\varepsilon_{\text {Ashby }}^{\prime}$ varies as a function of $\left(1 / d^{3}\right)$, and is expected to strongly increase with decreasing $d$. However, the experimental results reported for $n$-metal specimens ${ }^{3-8}$ showed a deviation from the prediction in theory. For example, Sanders et al. ${ }^{3}$ reported that the creep rates of $n-\mathrm{Cu}$ and $n-\mathrm{Pd}$ are slower by
2-4 orders of magnitude than the values calculated for the homogeneous GB diffusion creep model. Cai et al. ${ }^{4,5}$ reported that the observed creep rate of $n-\mathrm{Cu}$ is much lower than that expected from the homogeneous GB diffusion creep model, and then the observed activation enthalpy for the GB diffusion coefficient is considerably lower than that reported for coarse grained polycrystalline $\mathrm{Cu}$. On the other hand, Wang et al. ${ }^{6}$ and Yin and co-workers ${ }^{7,8}$ reported that the observed creep rates of $n$-Ni increase to the value predicted in the homogeneous GB diffusion creep model at the elevated applied stress $\sigma_{a p}$. It is suggested that the deviation from the prediction in theory is associated with the quality of $n$-metal specimens used, or is indicative of a deviation of the underlying creep mechanism from the theoretical model. For the elastic property of $n$ metals, although a very low elastic modulus has been reported in the pioneer works, ${ }^{9-11}$ recent elasticity studies on high-density $n$ metals ${ }^{12-16}$ indicated that such a low elastic modulus as that reported should mainly be associated with pores contained in $n$-metal specimens used. That is, the Young's modulus of the GB regions is found to be comparable to that of the corresponding bulk metal, indicating that the atomic density of the GB regions is comparable with the bulk value. Since recent creep studies ${ }^{3-8}$ have been made using the high-density $n$-metal specimens, the reported results presumably suggest that the plastic creep mechanism is a function of the applied stress $\sigma_{a p}$ different from Eq. (1). In the present work, in order to clarify the underlying plastic creep mechanism of $n$ metals, we carried 
out a systematic creep study of high-density $n$-metal specimens, here on the $n$-Au specimens. ${ }^{16-20}$

\section{EXPERIMENTS}

Nanocrystalline gold ( $n$-Au, hereafter) specimens were prepared using the gas deposition (GD) method. ${ }^{16-18}$ The present GD apparatus is composed of an evaporation chamber, a deposition chamber, a transfer pipe connecting these chambers, and a helium circulation system with purification columns. During an operation of the GD apparatus, a purified 99.9999\% helium gas, was supplied to the evaporation chamber, and the supplied helium gas was evacuated from the evaporation and deposition chambers to the helium circulation system with purification columns. Helium gas pressures in the evaporation and deposition chambers were 67 $\mathrm{kPa}$ and $133 \mathrm{~Pa}$, respectively. In the evaporation chamber, 99.9999\% purity Au filled in a graphite crucible was heated to $1600-1900 \mathrm{~K}$ by a rf-induction heater. Just above the Au melt, ultrafine $\mathrm{Au}$ particles were formed by a gas condensation process. The ultrafine Au particles were sucked by the transfer pipe and transferred onto a glass substrate in the deposition chamber. In order to avoid an undesirable heating of the glass substrate by a helium gas flow as a carrier of the ultrafine $\mathrm{Au}$ particles, the glass substrate was cooled by a cold finger to keep its temperature below $300 \mathrm{~K}$. It is found that the purity of the helium gas supplied to the evaporation chamber critically governs the quality of $n$-Au specimens obtained. The purity of the helium gas should be as high as $99.9999 \%$ in order to obtain contamination-free and fulldensity $n$-Au specimens. A grain size in the specimens was measured from the STM, indicating that the mean grain size was about $20 \mathrm{~nm}$ in the present $n$-Au specimens. X-raydiffraction measurements showed that the crystallographic directions of almost all the crystallites normal to the flat surface of the specimens was (111), suggesting that a certain spontaneous reorientation of ultrafine particles took place on the specimen surface. It is also found that a deposition rate plays a critical role on the property of $n$-Au specimens. ${ }^{18}$ The $n$-Au specimens prepared with deposition rates beyond $800 \mathrm{~nm} / \mathrm{s}$ (here 800-1900 nm/s) and those deposited below $800 \mathrm{~nm} / \mathrm{s}$ (here $200-700 \mathrm{~nm} / \mathrm{s}$ ) will be referred to as the type- $H$ and type- $L$ specimens below, respectively. The density of the $n$-Au specimens was $19.4 \pm 0.2 \mathrm{~g} / \mathrm{cm}^{3}$ for both the type- $H$ and type- $L$ specimens, which shows a good agreement with $19.32 \mathrm{~g} / \mathrm{cm}^{3}$ reported for the bulk $\mathrm{Au}$ value in literature (see Ref. 18 for details). On the other hand, an onset temperature for the crystalline growth is about $400 \mathrm{~K}$ for the type- $H$ specimens and about $500 \mathrm{~K}$ for the type- $L$ specimens, respectively. ${ }^{17,18}$ Further, it is found in the present work that a plastic creep behavior is different between the type- $H$ and type- $L$ specimens. We surmise that the spontaneous reorientation of ultrafine particles on the specimen surface is completed for the type- $L$ specimens but is not sufficient for the type- $H$ specimens, respectively.

A size of the $n$-Au specimens was $23 \mathrm{~mm}$ long and $1 \mathrm{~mm}$ wide. The thickness of the $n$-Au specimens was about 20 $\mu \mathrm{m}$ for specimens used for tensile tests and about $500 \mu \mathrm{m}$ for specimens used for compressive tests. The tensile creep

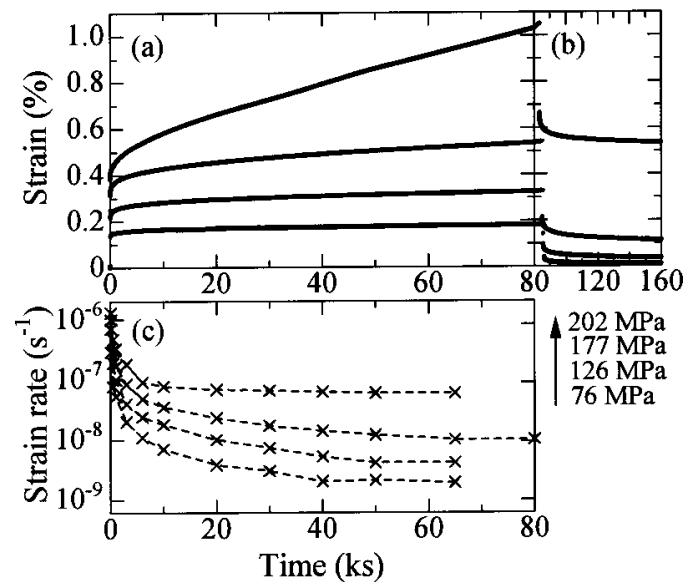

FIG. 1. (a) Examples of the tensile creep curves measured under $\sigma_{a p}$ of $76,126,177$, and $202 \mathrm{MPa}$. (b) Elastic aftereffect curves after unloading. These measurements were sequentially made at 295 $\mathrm{K}$ for the type- $H$ specimen. (c) Differential curves of the creep curves shown in (a).

tests were made in a temperature range between 250 and 360 $\mathrm{K}$, and the compressive creep tests were carried out at 295 and $320 \mathrm{~K}$, respectively. We constructed a tensile creep test apparatus designed for a thin tape specimen and the compressive creep test apparatus for $500-\mu$ m-thick specimens, where the creep strains were measured using an capacitance displacement sensor. The sensitivity of the sensor was $10 \mathrm{~nm}$ for the tensile test apparatus and $5 \mathrm{~nm}$ for the compressive test apparatus, respectively. It is noted that the compressive tests were not done for the type- $L$ specimens because the deposition rate was too low to prepare a thick specimen in a reasonable deposition time.

The cross section and gauge length of specimens used for the tensile creep tests are $2 \times 10^{-2} \mathrm{~mm}^{2}$ and $15-20 \mathrm{~mm}$, respectively. The cross section and the gauge length of specimens used for the compressive creep tests were 2 $\times 10^{-1} \mathrm{~mm}^{2}$ and $500 \mu \mathrm{m}$, respectively, after mechanical polishing of the specimen surfaces. In order to study the creep property near the yield stress, indentation creep tests were carried out at room temperature by using the microVicker's hardness tester. A change in the surface morphology of $n$-Au specimens due to creep deformations was checked by scanning tunneling microscopy (STM).

\section{RESULTS}

\section{A. Macroscopic creep behaviors}

Figure 1(a) shows examples of tensile creep curves observed in the stress range between 76 and $202 \mathrm{MPa}$, and Fig. 1(b) shows elastic aftereffect curves after unloading, where the creep tests for $80 \mathrm{ks}$ and the subsequent elastic aftereffect measurements were sequentially made with the increase in the applied stress, $\sigma_{a p}$, at $295 \mathrm{~K}$ for the type- $H$ specimen. As seen in Fig. 1(b) a strain found just after unloading, $\varepsilon_{80 \mathrm{ks}}$, is composed of the anelastic strain and the plastic creep strain. The anelastic strain increases linearly with the increase in $\sigma_{a p}$ for $\sigma_{a p}$ beyond a few $\mathrm{MPa}$, and is associated with a certain anelastic process in the grain-boundary regions. ${ }^{17}$ The 


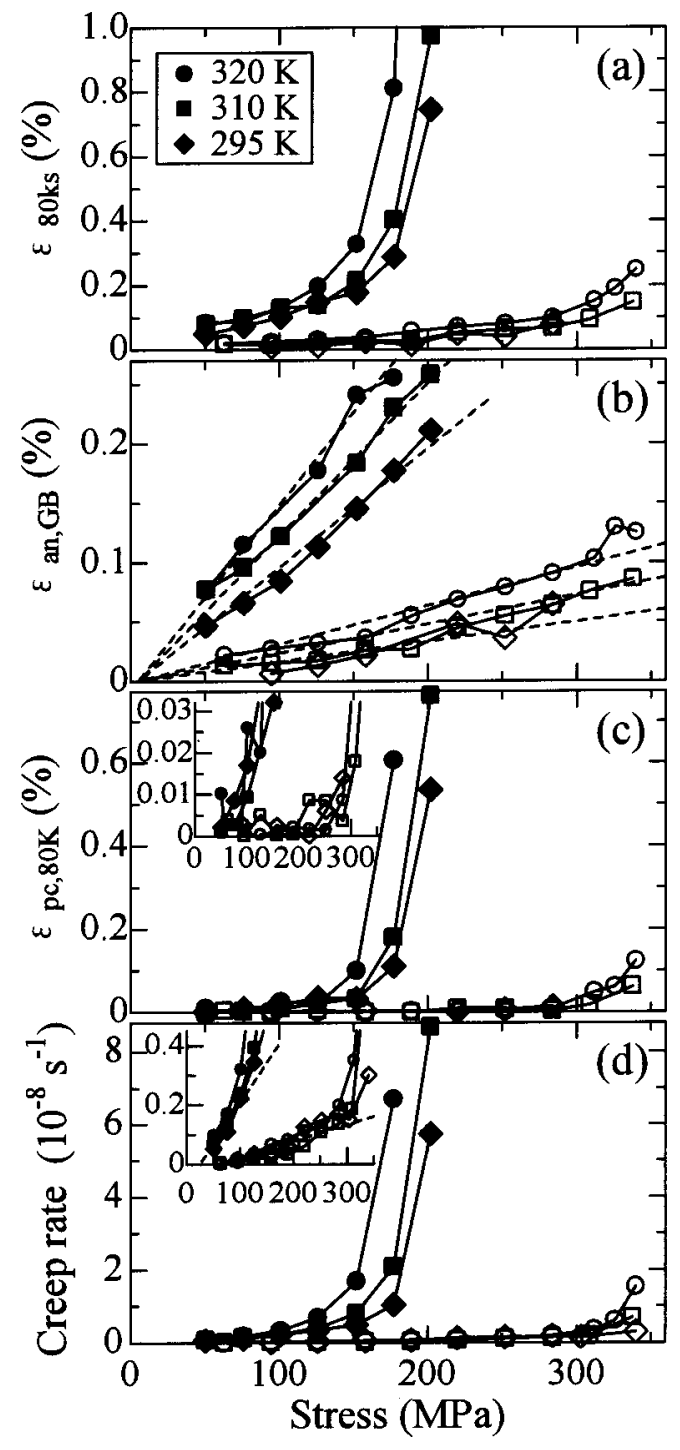

FIG. 2. (a) The $\varepsilon_{80 k s}$ vs $\sigma_{a p}$ data observed at 295, 310, and 320 $\mathrm{K}$ for the type- $H$ specimens (filled symbols) and the type- $L$ specimens (open symbols). (b)-(d) show the data for $\varepsilon_{a n, \mathrm{~GB}}$ and $\varepsilon_{p c}$, and the creep rate found after a loading time for $80 \mathrm{ks}$, respectively. See the text for $\varepsilon_{80 k s}, \varepsilon_{a n, \mathrm{~GB}}, \varepsilon_{p c}$, and $\sigma_{a p}$.

anelastic strain will be referred to as $\varepsilon_{a n, \mathrm{~GB}}$ below. It is also seen in Fig. 1(b) that the plastic creep strain, $\varepsilon_{p c, 80 k s}$, attained after loading for $80 \mathrm{ks}$ is much smaller than $\varepsilon_{a n, \mathrm{~GB}}$ for a $\sigma_{a p}$ of $76 \mathrm{MPa}$ and much larger than $\varepsilon_{a n, \mathrm{~GB}}$ for a $\sigma_{a p}$ of $202 \mathrm{MPa}$. Figure 1(c) shows differential curves of the tensile creep curves shown in Fig. 1(a), where $\varepsilon_{a n, \mathrm{~GB}}$ shows saturation and a steady creep is attained after about $50 \mathrm{ks}$ in the present creep tests.

Figure 2(a) shows $\varepsilon_{80 k s}$ as a function of $\sigma_{a p}$, were observed at 295,310 , and $320 \mathrm{~K}$ for the type- $H$ and type- $L$ specimens. Figure $2(\mathrm{~b})-2(\mathrm{~d})$ show $\varepsilon_{a n, \mathrm{~GB}}, \varepsilon_{p c, 80 k s}$, and the steady creep rate $\varepsilon^{\prime}$, found near $80 \mathrm{ks}$ as a function of $\sigma_{a p}$, respectively. As already mentioned, $\varepsilon_{a n, \mathrm{~GB}}$ increases linearly with the increase in $\sigma_{a p}$ for $\sigma_{a p}$ beyond a threshold stress $\sigma_{a n 1}$ of a few MPa. The ratio of $\varepsilon_{a n, \mathrm{~GB}}$ to the elastic strain, $\varepsilon_{e 1}$, is as large as 1.1 at $320 \mathrm{~K}$ and 0.7 at $295 \mathrm{~K}$ for the

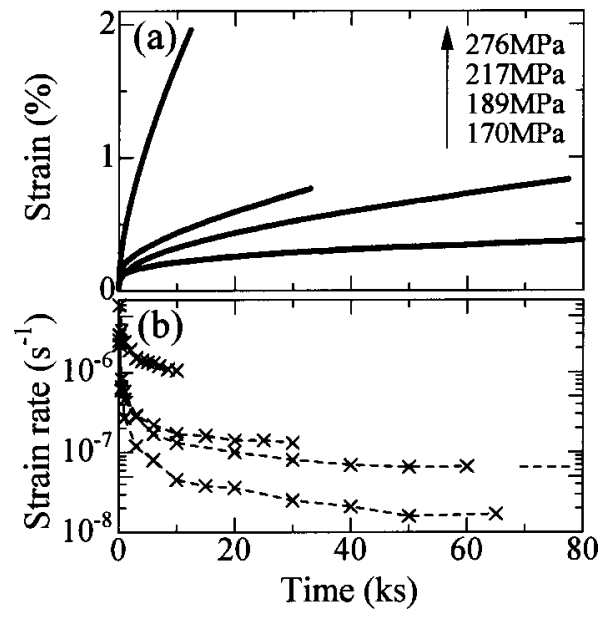

FIG. 3. (a) Examples of the compressive creep curves under various $\sigma_{a p}, 170,189,217$, and $276 \mathrm{MPa}$, observed for the type- $H$ specimen at 295 K. (b) Differential curves of the creep curves shown in (a).

type- $H$ specimens and 0.2 at $320 \mathrm{~K}$ and 0.1 at $295 \mathrm{~K}$ for the type- $L$ specimens. Further the ratio $\varepsilon_{a n, \mathrm{~GB}} / \varepsilon_{e 1}$ decreases with decreasing temperature (this issue will be mentioned later in Fig. 11). As seen in Figs. 2(c) and 2(d), the plastic creep strain can be detected for $\sigma_{a p}$ beyond the threshold applied stress $\sigma_{p c 1}$, which is about 30 and $60 \mathrm{MPa}$ for the type- $H$ and type- $L$ specimens, respectively. As seen in Fig. $2(\mathrm{~d}), \varepsilon^{\prime}$ increases almost linearly with the increase in $\sigma_{a p}$ for $\sigma_{a p}$ beyond $\sigma_{p c 1}$, and then shows a steep increase for $\sigma_{a p}$ beyond the threshold applied stress $\sigma_{p c 2}$, which is about 150 and $300 \mathrm{MPa}$ for type $H$ and type- $L$ specimens, respectively.

Figure 3(a) shows examples of the compressive creep curves observed for the type- $H$ specimens at $295 \mathrm{~K}$, which were conducted to pursue the creep response at high stresses. Figure 3(b) shows differential curves of the creep curves shown in Fig. 3(a). The creep tests at 217 and $276 \mathrm{MPa}$ were interrupted at about 33 and $12 \mathrm{ks}$, respectively, where the creep rate appeared to attain its steady value, and the steady creep rate was estimated as the minimum creep rate.

It is reported that the micro-Vicker's hardness tests may give an insight into the creep response at very high stresses. $^{21}$ The mean creep rate can be estimated from the hardness $H$, the hardness change rate $H^{\prime}$, and the material constant $\kappa$, by using the relationship,

$$
\varepsilon^{\prime}=6 /(1+\kappa)^{3} H^{\prime} / H .
$$

$\kappa$ is given as $c / a$, where $a$ and $c$ are the half-length of the diagonal line of an indenture and the half-width of the elastic-plastic zone around the indenture, respectively. A value of $\kappa$ is found between 2 and 3 for most of polycrystalline metals. We conducted the micro-Vicker's hardness tests with loading of $9.8 \times 10^{-2} \mathrm{~N}$ for various loading times at $295 \mathrm{~K}$ for the type- $H$ and type- $L$ specimens. Values of the hardness found after loading times of 5, 30, and $520 \mathrm{~s}$, will be referred to as $H_{5 s}, H_{30 s}$ and $H_{520 s}$ below, respectively. We estimated the yield stress $\sigma_{y}$ from a value of $H_{5 s}$ using the well known relationship of $\sigma_{y} \approx H / 3$ and $H^{\prime}$ from a change in the values of $H_{30 s}$ and $H_{520 s}$. We found $H_{5 s}$ 


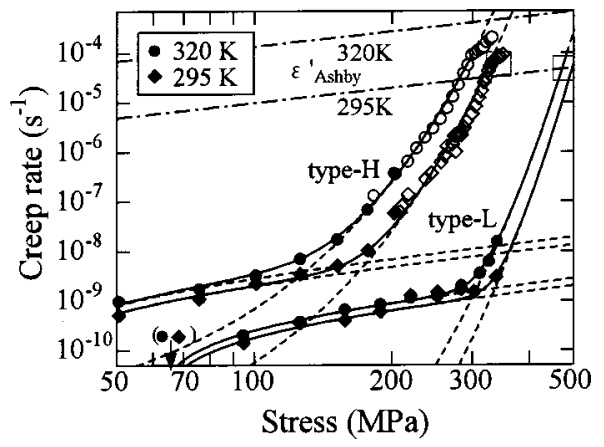

FIG. 4. The $\varepsilon^{\prime}$ vs $\sigma_{a p}$ data observed for the type- $H$ and $L$ specimens at 295 and $320 \mathrm{~K}$. The filled symbols denote the tensile creep rate data, the open symbols show the compressive creep test data, and the open squares denote the data estimated from the micro-Vicker's hardness tests. The dashed lines fitted to the region I data and the region II data denote $\varepsilon_{I}^{\prime}$ estimated by using Eq. (3) and $\varepsilon_{I I}^{\prime}$ estimated by using Eq. (4), respectively. The solid curves fitted to region I and II data are $\varepsilon_{I}^{\prime}+\varepsilon_{I I}^{\prime}$. $\varepsilon_{\text {Ashby }}^{\prime}$ at 295 and $320 \mathrm{~K}$ estimated for $d=20 \mathrm{~nm}$ at 295 and $320 \mathrm{~K}$ are also shown. See the text for details.

$=1.1 \mathrm{GPa}$ and $H^{\prime} / H=3.1( \pm 0.1) \times 10^{-4} \mathrm{~s}^{-1}$ for the type- $H$ specimens and $H_{5 s}=1.5 \mathrm{GPa}$ and $H^{\prime} / H=2.7( \pm 0.1)$ $\times 10^{-4} \mathrm{~s}^{-1}$ for the type- $L$ specimens, respectively. These values give $\sigma_{y}=360 \mathrm{MPa}$ and $\varepsilon^{\prime}=7$ to $3 \times 10^{-5} \mathrm{~s}^{-1}$ in the stress range between 320 and $360 \mathrm{MPa}$ for the type- $H$ specimens, and $\sigma_{y}=500 \mathrm{MPa}$ and $\varepsilon^{\prime}=6$ to $3 \times 10^{-5} \mathrm{~s}^{-1}$ in the stress range between 440 and $500 \mathrm{MPa}$ for the type- $L$ specimens, respectively.

Figure 4 shows the creep rate $\varepsilon^{\prime}$ data as a function of the applied stress $\sigma_{a p}$, where the data found for the tensile creep tests, the compressive creep tests and the micro-Vicker's hardness tests are compared. As seen in Fig. 4, the $\varepsilon^{\prime}$ vs $\sigma_{a p}$ data show a letter S-like curve for both type- $H$ and type- $L$ specimens. We shall classify the observed creep response into three categories as follows: Region I: the linear creep rate $\varepsilon_{p c 1}^{\prime}$ region found for $\sigma_{a p}$ between $\sigma_{p c 1}$ and $\sigma_{p c 2}$. Region II: the transient creep rate $\varepsilon_{p c \text { II }}^{\prime}$ region found for $\sigma_{a p}$ between $\sigma_{p c 2}$ and $\sigma_{p c 3}$. Region III: the saturation creep rate, $\varepsilon_{p c \text { III }}^{\prime}$, region found for $\sigma_{a p}$ between $\sigma_{p c 3}$ and $\sigma_{y}$, where $\sigma_{p c 1}, \sigma_{p c 2}$, and $\sigma_{y}$ are about 30,150 , and $360 \mathrm{MPa}$ for the type- $H$ specimens, and about 60,300 , and $500 \mathrm{MPa}$ for the type- $L$ specimens, respectively. $\sigma_{p c 3}$ is slightly lower than $\sigma_{y}$.

As already mentioned, the Ashby creep rate $\varepsilon_{\text {Ashby }}^{\prime}$ for the homogeneous GB diffusion creep is given by Eq. (1). For the application of Eq. (1) to the present creep data, we assume that $D_{\mathrm{GB}, 0}$ and $E_{\mathrm{GB}}$ for the $n$-Au specimens are 6.2 $\times 10^{-7} \mathrm{~m}^{2} \mathrm{~s}^{-1}$ and $0.88 \mathrm{eV}$ reported for coarse-grained polycrystalline gold, ${ }^{22}$ respectively, and $\delta$ is $0.5 \mathrm{~nm}$. $\Gamma$ $=0.2( \pm 0.1) \mathrm{J} / \mathrm{m}^{2}$ reported for the type- $H$ specimens ${ }^{18}$ gives $\sigma_{0, \text { Ashby }}$ of several MPa for $d=20 \mathrm{~nm}$. Then one may find that $\varepsilon_{\text {Ashby }, 0}^{\prime}$ for $d=20 \mathrm{~nm}$ is $2.2 \times 10^{-19} \mathrm{~s}^{-1} \mathrm{~Pa}^{-1} \mathrm{~J}^{-1}$ and $\varepsilon_{\text {Ashby }}^{\prime}$ for $d=20 \mathrm{~nm}$ is as large as $4.9 \times 10^{-14} \sigma \mathrm{s}^{-1}$ at $295 \mathrm{~K}$ and $6.8 \times 10^{-13} \sigma \mathrm{s}^{-1}$ at $320 \mathrm{~K}$ where $\sigma$ is measured in $\mathrm{Pa}$ and $\sigma_{0, \text { Ashby }}$ is neglected. In Fig. $4, \varepsilon_{\text {Ashby }}^{\prime}$ for $d=20 \mathrm{~nm}$ at 295 and $320 \mathrm{~K}$ are shown by straight lines, where $\varepsilon_{\text {Ashby' }}$

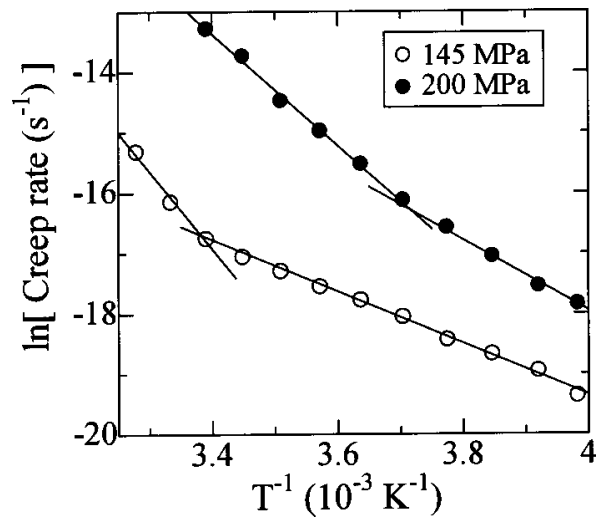

FIG. 5. Examples of the Arrhenius plot for $\varepsilon^{\prime}$ in regions I and II, where the $\varepsilon^{\prime}$ data were observed in the tensile creep tests at 145 and $200 \mathrm{MPa}$ for the type- $H$ specimen.

appears to explain the $\varepsilon^{\prime}$ vs $\sigma_{a p}$ data found in region III. The creep rate observed in region III will be referred to as $\varepsilon_{p c \text { III }}^{\prime}$. In order to estimate the activation enthalpy for the plastic creep in region I, $E_{p c \mathrm{I}}$ and region II, $E_{p c \text { II }}$, we tentatively assumed that the creep rate observed in region I, $\varepsilon_{p c \mathrm{I}}^{\prime}$, and that in region II, $\varepsilon_{p c \text { II }}^{\prime}$, are respectively explained by the following empirical expressions:

$$
\begin{gathered}
\varepsilon_{p c \mathrm{I}}^{\prime}=\varepsilon_{p c \mathrm{I}, 0}^{\prime}\left(\sigma_{a p}-\sigma_{p c 1}\right) \exp \left(-E_{p c \mathrm{I}} / k T\right) \\
\text { for } \sigma_{p c 1} \leqslant \sigma_{a p}<\sigma_{p c 2}
\end{gathered}
$$

and

$$
\begin{gathered}
\varepsilon_{p c \mathrm{II}}^{\prime}=\varepsilon_{p c \mathrm{II}, 0}^{\prime} \exp \left[C_{p c \mathrm{II}}\left(\sigma_{a p}-\sigma_{p c 2}\right)\right] \exp \left(-E_{p c \mathrm{II}} / k T\right) \\
\text { for } \sigma_{p c 2} \leqslant \sigma_{a p}<\sigma_{p c 3} .
\end{gathered}
$$

It is noted that a value of the creep rate in region I is lower by four or five orders than $\varepsilon_{\text {Ashby }}^{\prime}$ estimated for $d=20 \mathrm{~nm}$. The term $\exp \left[C_{p c I I}\left(\sigma_{a p}-\sigma_{p c 2}\right)\right]$ in Eq. (4) is tentatively assumed to explain the steep increase in $\varepsilon^{\prime}$ with the increase in $\sigma_{a p}$, where $C_{p c \text { II }}$ is a constant. Figure 5 shows the Arrhenius plot for $\varepsilon^{\prime}$ in regions I and II, where $E_{p c \mathrm{I}}$ found is 0.4 $\pm 0.1 \mathrm{eV}$ for both the type- $H$ and type- $L$ specimens, and $E_{p c \text { II }}$ found is $1.2 \pm 0.1$ and $1.3 \pm 0.1 \mathrm{eV}$ for the type- $H$ and type- $L$ specimens, respectively.

\section{B. Microscopic creep textures}

To pursue an insight into the creep mechanism of $n-\mathrm{Au}$, we monitored a change in the surface morphology due to a plastic creep deformation using STM. Figure 6 is an example of the STM image for the type- $L$ specimen before the creep test, indicating that the specimen surface is smooth and flat except that grains can be recognized as the network contour. It is noted that the surface morphology for both the type- $H$ and type- $L$ specimens is very similar to that shown in Fig. 6 . Figures 7(a) and 7(b) show STM images of the type- $H$ specimen after the plastic creep deformation by $0.3 \%$ at $295 \mathrm{~K}$ under $\sigma_{a p}$ of $100 \mathrm{MPa}$ in region I where Fig. 7(a) is an example of most of the specimen surface and Fig. 7(b) shows a mesoscopic scale GB slip observed here and there with a low areal density. It is not shown here, but STM images of 


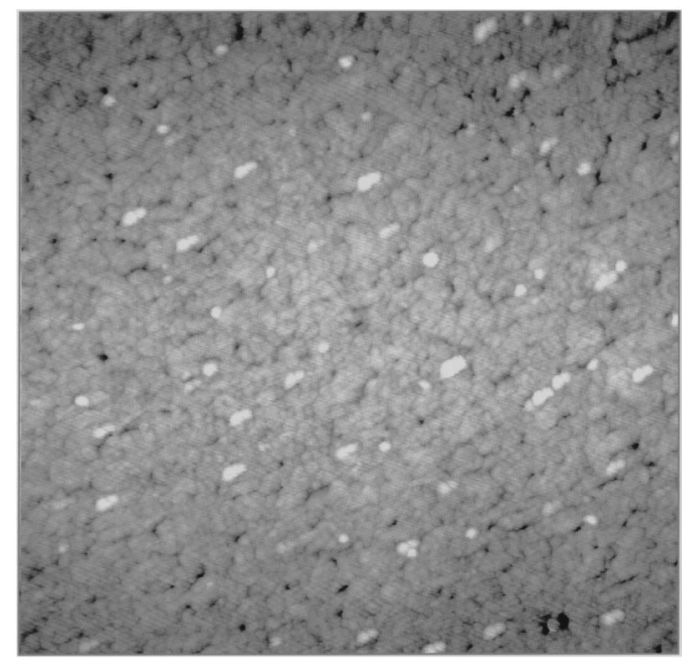

FIG. 6. An example of the STM images observed for the type- $L$ specimen before the creep test. The scanning area is 1000 $\times 1000 \mathrm{~nm}^{2}$, and the black to white contrast measures a change in height as $5 \mathrm{~nm}$.

the type- $L$ specimen after the plastic creep deformation in region I are very similar to those shown in Figs. 7(a) and 7(b). STM observations indicate that the localized mesoscopic scale GB slip takes place during the plastic creep deformation in region I and the plastic creep deformation mechanism in region I is very similar between the type- $H$ and type- $L$ specimens.

Figures 8(a)-8(c) show examples of STM images observed after tensile creep tests in region II: Figs. 8(a) and 8 (c) for the type- $H$ specimen after the plastic creep deformation by $2 \%$ at $295 \mathrm{~K}$ under $170 \mathrm{MPa}$, and Fig. 8 (b) for the type- $L$ specimen after the plastic creep deformation by $4 \%$ at $330 \mathrm{~K}$ under $350 \mathrm{MPa}$, respectively. The STM images seen Figs. 8(a)-8(c) are very similar to the STM image shown in Fig. 7(b) except that the mean separation between the localized GB slips seen in Figs. 8(a)-8(c) is much shorter than that seen in Fig. 7(b), and the areal density of the localized GB slips seen in Figs. 8(a)-8(c) is much higher than that seen in Fig. 7(b). In Figs. 8(a)-8(c), the length and step height of the localized GB slips are between several tens and a few hundred $\mathrm{nm}$ and about $1 \pm 1 \mathrm{~nm}$ respectively. From the
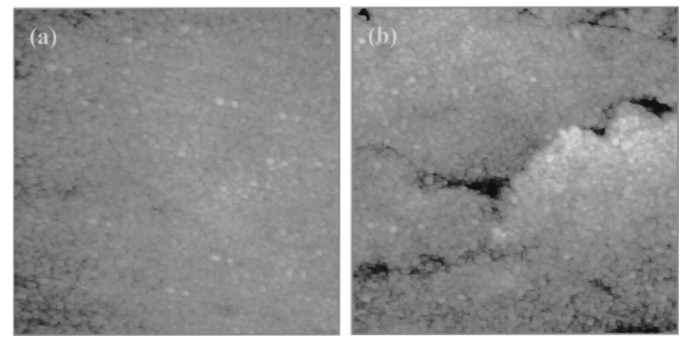

FIG. 7. Examples of the STM images observed for the type- $H$ specimen after plastic creep deformation by $0.3 \%$ at $295 \mathrm{~K}$ under $100 \mathrm{MPa}$ in region I. (a) Most of the specimen surface, (b) A mesoscopic scale GB slip observed here and there, but the areal density is very low. The scanning area is $1000 \times 1000 \mathrm{~nm}^{2}$, and the black to white height is $6 \mathrm{~nm}$ for (a) and $11 \mathrm{~nm}$ for (b), respectively.
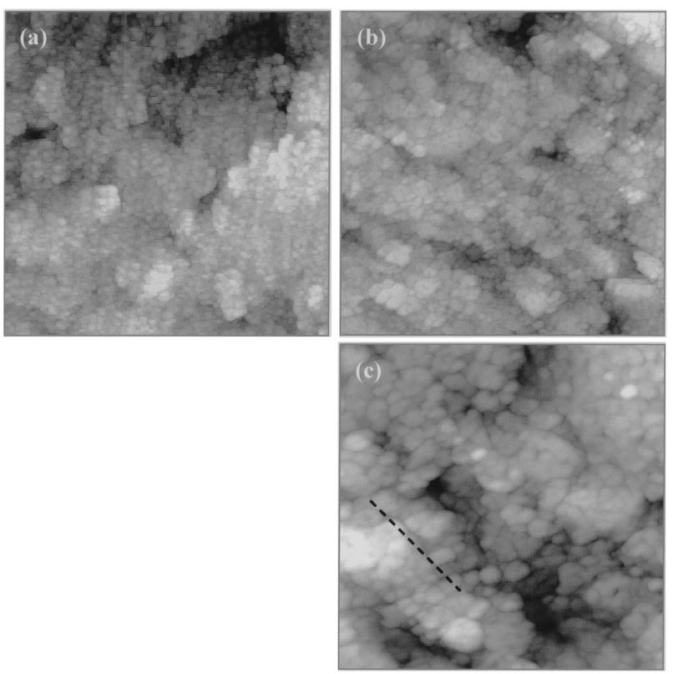

FIG. 8. Examples of the STM images observed for the type- $H$ and the $L$ specimens after the tensile creep tests in region II. (a) and (c) show the type- $H$ specimen after plastic creep deformation by $2 \%$ at $295 \mathrm{~K}$ under $170 \mathrm{MPa}$. (b) Shows the type- $L$ specimen after plastic creep deformation by $4 \%$ at $320 \mathrm{~K}$ under $350 \mathrm{MPa}$. The scanning area and the black to white height are $1000 \times 1000 \mathrm{~nm}^{2}$ and $5 \mathrm{~nm}$ for (a), $1000 \times 1000 \mathrm{~nm}^{2}$ and $12 \mathrm{~nm}$ for (b), and 500 $\times 500 \mathrm{~nm}^{2}$, and $7 \mathrm{~nm}$ for $(\mathrm{c})$.

general feature of the STM images, it seems that the mean inclination angle of the localized GB slips from the tensile direction is about $45^{\circ}$. A typical example can be seen in Fig. 8(c). The plastic creep strain $\varepsilon_{p c}$ associated with the localized GB slips may be estimated by the relationship

$$
\varepsilon_{p c} \approx(1 / L)^{3} \Delta d l^{2},
$$

where $\Delta d, l$ and $L$ are the mean slip displacement, the mean length of the localized GB slips, and the mean separation between the localized GB slips, respectively. The application of Eq. (5) to the case with the values of $\Delta d=1 \mathrm{~nm}, l$ $=200 \mathrm{~nm}$, and $L=100 \mathrm{~nm}$, which are roughly estimated from Figs. 8 (a) and 8 (b), gives $\varepsilon_{p c} \approx 4 \%$, which is comparable to $\varepsilon_{p c} \approx 2 \%$ for Figs. 8 (a) and 8(c) and $\varepsilon_{p c} \approx 4 \%$ for Fig. 8(b). It is indicated that the localized GB slips are responsible for the plastic creep deformation in region II. Figures 9(a) and 9(b) show STM images observed for the type- $H$ specimen after the tensile creep test at $295 \mathrm{~K}$ with the increased $\sigma_{a p}$ of $200 \mathrm{MPa}$ in region II, where the plastic creep deformation attained was $10 \%$. The observed morphology suggests that crossing of the localized GB slips takes place. STM images observed for the type- $L$ specimens were very similar to those shown in Figs. 9(a) and 9(b) (not shown here).

Figures 10(a)-10(c) show examples of STM images observed for the type- $H$ and type- $L$ specimens after the creep tests in region III, which were made by the micro-Vicker's hardness tests (see Fig. 4). The indentations were done at 295 $\mathrm{K}$ for $1 \mathrm{ks}$ under a load of $0.98 \mathrm{~N}$, and then, after unloading, STM observations were made for the specimen surface nearby the indenture, at $2-20 \mu \mathrm{m}(c / a=1.1-1.5)$ from the edges of the indenture. The creep strain of the observed 


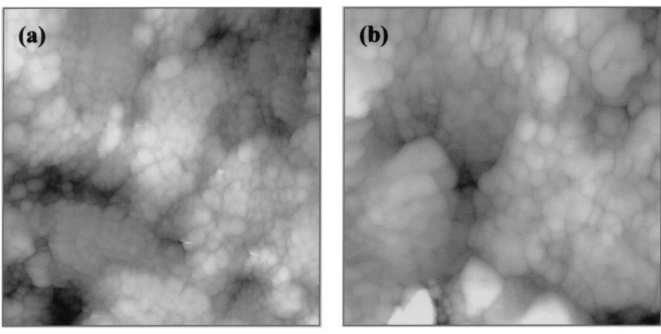

FIG. 9. Similar to Figs. 8(a) and 8(c) but here the STM images observed for the type- $H$ specimen after the tensile creep test at 295 $\mathrm{K}$ with the increased $\sigma_{a p}$ of $200 \mathrm{MPa}$ in region II are shown, where the plastic creep deformation attained was $10 \%$. The scanning area and the black to white height are $1000 \times 1000 \mathrm{~nm}^{2}$ and $25 \mathrm{~nm}$ for (a) and $500 \times 500 \mathrm{~nm}^{2}$ and $12 \mathrm{~nm}$ for (b), respectively.

specimen surface was estimated as several percent from the application of Eq. (1). It can be seen in Figs. 10(a)-10(c) that the mean separation between the localized GB slips decreases to the comparable scale with the grain sizes.

\section{DISCUSSION}

We found the following for the creep behaviors of the $n$-Au specimens.

(1) The strong anelastic strain $\varepsilon_{a n, \mathrm{~GB}}$ is revealed when the applied stress $\sigma_{a p}$ is increased beyond the threshold value of a few MPa, $\sigma_{a n 1}{ }^{17}$

(2a) The plastic creep response of the $n$-Au specimens is classified into three categories, regions I-III which are characterized by the threshold applied stresses $\sigma_{p c 1}-\sigma_{p c 3}$.

(2b) The localized GB slip is responsible for the plastic creep deformation.
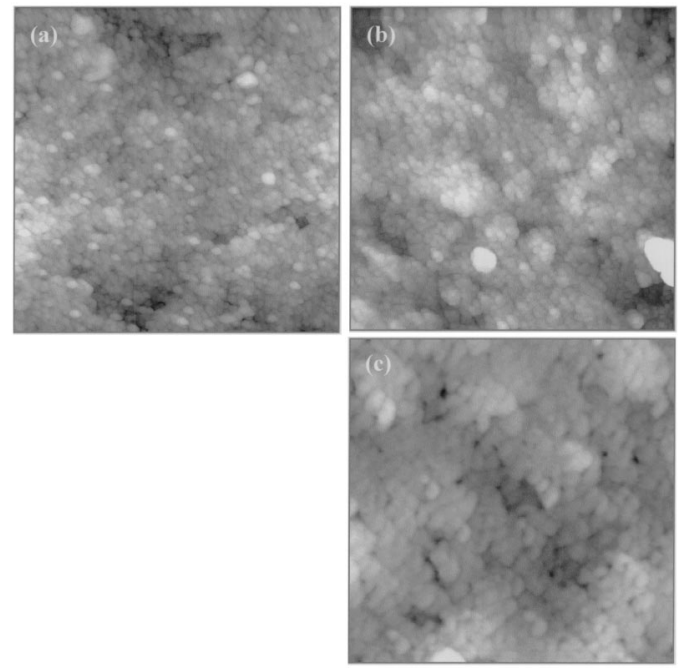

FIG. 10. Examples of the STM images observed after the creep tests in region III, which were made by the micro-Vicker's hardness, (a) for the type- $H$ specimen, and (b) and (c) for the type- $L$ specimen. The scanning area and the black to white height are $1000 \times 1000 \mathrm{~nm}^{2}$ and $5 \mathrm{~nm}$ for (a), $1000 \times 1000 \mathrm{~nm}^{2}$ and $7 \mathrm{~nm}$ for (b), and $500 \times 500 \mathrm{~nm}^{2}$ and $7 \mathrm{~nm}$ for (c), respectively. See the text for testing conditions.

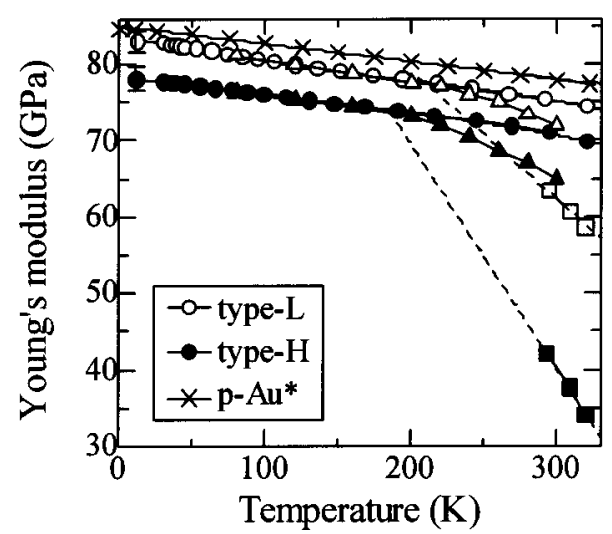

FIG. 11. The Young's modulus vs temperature data. The filled symbols for the type- $H$ specimens and the open symbols for the type- $L$ specimens. The circular symbols are the data observed in the vibrating reed measurements at $10^{2} \mathrm{~Hz}$ with a strain amplitude of $10^{-6}$. The triangular symbols are the data observed in the tensile tests at a strain rate of $4.5 \times 10^{-4} / \mathrm{s}$ in a strain range between $10^{-4}$ and $10^{-3}$ (Ref. 3). The crosses are the data for the coarse-grained $p$-Au. (Ref. 16). The squares denote the Young's modulus estimated from the $\varepsilon_{a n, \mathrm{~GB}}$ data shown in Fig. 2(b). See the text for details.

(2c) No hardening and no softening are detected for the plastic creep deformation.

(2d) The mean separation between the localized GB slips in region II decreases with the increase in the applied stress $\sigma_{a p}$.

Item (1) will be discussed first. Figure 11 shows the temperature dependence of the Young's modulus $M_{R}$, observed for type- $H$ specimens and type- $L$ specimens, where we show data observed in the vibrating reed measurements at $10^{2} \mathrm{~Hz}$ with a strain amplitude of $10^{-6}$ and those observed in tensile tests at a strain rate of $4.5 \times 10^{-4} / \mathrm{s}$ in a strain range between $10^{-4}$ and $10^{-3}$. The Young's modulus reported for the coarse-grained polycrystalline (p) Au (Ref. 22) is also shown. The temperature dependence of the Young's modulus of the type- $H$ and type- $L$ specimens shows a parallel change with that of the coarse grained $p$-Au except that the Young's modulus of the type- $H$ and type- $L$ specimens shows a decrease from the parallel change above $200 \mathrm{~K}$. The decrease from the parallel change above $200 \mathrm{~K}$ is associated with $\varepsilon_{a n, \mathrm{~GB}} \cdot{ }^{17}$ As mentioned in Fig. 2(b), one may find the relationship between $\varepsilon_{a n, \mathrm{~GB}}$ and $\sigma_{a p}$ in the high $\sigma_{a p}$ range between 50 and $200 \mathrm{MPa}$ for the type- $H$ specimens, and between 50 and $350 \mathrm{MPa}$ for the type- $L$ specimens, as

$$
\varepsilon_{a n, \mathrm{~GB}}=J_{a n, \mathrm{~GB}}\left(\sigma_{a p}-\sigma_{a n 1}\right),
$$

where $J_{a n, \mathrm{~GB}}$ is the anelastic compliance, i.e., the linear anelasticity for $\sigma_{a p}$ beyond $\sigma_{a n 1}$. We evaluated $\varepsilon_{a n, \mathrm{~GB}}$ from the data shown in Fig. 2(b) and then estimated $M_{R}$ using the relationship

$$
M_{R} \approx\left[\varepsilon_{e 1} /\left(\varepsilon_{e 1}+\varepsilon_{a n, \mathrm{~GB}}\right)\right] M_{0},
$$

where $M_{0}$ is the Young's modulus observed in the vibrating reed measurements. The $M_{R}$ data shown in Fig. 11 indicate that the elastic compliance $J_{e 1}$, in the the relationship between $\varepsilon_{e 1}$ and $\sigma_{a p}$, 


$$
\varepsilon_{e 1}=J_{e 1} \sigma_{a p},
$$

is smaller than $J_{a n, \mathrm{~GB}}$. It is noted that $J_{a n, \mathrm{~GB}}$ is much smaller than $J_{e 1}$ in $p$ metals. Further, for the $M_{R}$ data shown in Fig. 11, the empirical relationship between $\varepsilon_{a n, \mathrm{~GB}}$ and $T$ may be found as

$$
\varepsilon_{a n, \mathrm{~GB}}=C_{a n, \mathrm{~GB}}\left(T-T_{a n 1}\right),
$$

where $C_{a n, \mathrm{~GB}}$ is the temperature coefficient of $\varepsilon_{a n, \mathrm{~GB}}$, and the onset temperature $T_{a n 1}$ is about $200 \mathrm{~K}$ or $0.15 T_{m}$, when $T_{m}$ is the melting temperature. The empirical relationship between $\varepsilon_{a n, \mathrm{~GB}}$ and $T$ may not be explained by the Arrhenius relationship, and this issue will be mentioned later. On the other hand, Bonetti et al. ${ }^{23}$ reported that a strong stress relaxation of $10 \%$ for $n$-Fe specimens at $0.17 T_{m}$ and one of $6 \%$ for $n$-Ni at $0.18 T_{m}$ are observed in the as-prepared state. They also reported that an amount of the stress relaxation decreases to a few percent after annealing at $473 \mathrm{~K}$, although no grain growth is detected after the annealing. The anelastic responses observed for $n$ - $\mathrm{Ni}$ and $n$-Fe are very similar to that for $\varepsilon_{a n, \mathrm{~GB}}$ in the present $n$-Au.

The activation enthalpy $E_{a n, \mathrm{~GB}}$ for the early stage of the GB anelasticity was studied in a previous stress relaxation experiment, ${ }^{18}$ where $E_{a n, \mathrm{~GB}}$ was found to be $0.2 \mathrm{eV}$, much lower than the activation enthalpy of $0.88 \mathrm{eV}$ reported for the GB diffusion in $p$-Au. ${ }^{22} T_{a n 1}$ of $200 \mathrm{~K}$ is in the temperature range where an atomic migration is already stimulated for a simple diffusion process with an activation enthalpy of 0.2 $\mathrm{eV}$, suggesting that a value of the pre-exponential factor for the atomic motion responsible for $\varepsilon_{a n, \mathrm{~GB}}$ should be very low. It is noted that recent molecular dynamics simulation studies for the GB diffusion ${ }^{24}$ and the migration ${ }^{25,26}$ reported that cooperative motions of many atoms can be excited above a certain temperature, which results in a low activation enthalpy and a low pre-exponential factor. We surmise that such a cooperative atomic motion of many atoms is responsible for $\varepsilon_{a n, \mathrm{~GB}}$ in the present $n$-Au. Then empirical relationship [Eq. (9)] suggests that the onset temperature for the cooperative motion of many atoms shows a distribution depending on the number of atoms associated. Further speculation is premature without more experiments.

We shall discuss the plastic creep response in the region I below. It is noted that $E_{p c \text { I }}$ found for both the type- $H$ and type- $L$ specimens is $0.4 \pm 0.1 \mathrm{eV}$, which is slightly higher than $E_{a n, \mathrm{~GB}}$ of $0.2 \mathrm{eV}$ and considerably lower than the activation enthalpy of $0.88 \mathrm{eV}$ reported for the $\mathrm{GB}$ diffusion in $p$-Au. ${ }^{22}$ Then we surmise that the cooperative motions of many atoms in the GB regions suggested in the moleculardynamics simulation studies ${ }^{24}$ can be developed to the localized GB slip at the elevated $\sigma_{a p}$. As already mentioned, the value of the creep rate $\varepsilon_{p c \text { I }}^{\prime}$ in region I is lower by four or five orders than $\varepsilon_{\text {Ashby }}^{\prime}$ estimated for $d=20 \mathrm{~nm}$, suggesting that not all the GB regions are associated with the plastic creep response in region I. The STM images shown in Fig. 7 indicate that only a minor part of the GB regions contributes to the plastic creep response, resulting in the very low creep rate. The reason why the localized GB slip is persistent, as seen in Fig. 7(b), may be due to the existence of $\sigma_{p c 1}$ and no work hardening for the localized GB slip is activated.
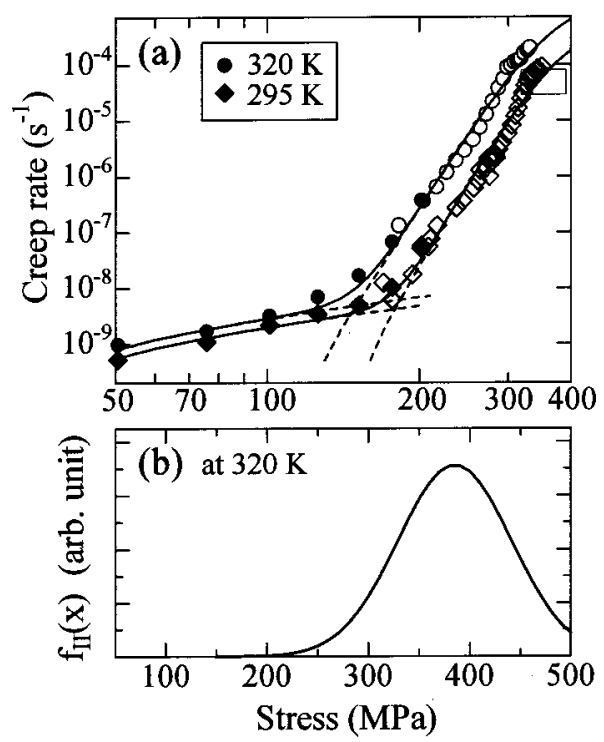

FIG. 12. (a) The best fitting of Eqs. (3) and (10) (the solid curves) to the creep rate vs stress data at $295 \mathrm{~K}$ and those at $320 \mathrm{~K}$ observed for the type- $H$ specimen. (b) The Gaussian distribution function $f_{I I}(x)$ found for the creep rate data at $320 \mathrm{~K}$ shown in (a).

The STM images shown in Figs. 8-10, observed in regions II and III can be explained by assuming a broad distribution for $\sigma_{p c 2}$ in region II, and assuming that the mean separation between the localized GB slips decreases to the size of each grain in region III. The empirical expression [Eq. (4)] for $\varepsilon_{p c \text { II }}^{\prime}$ suggests that the distribution of $\sigma_{p c 2}$ should be broad when the view mentioned above is the case. Here we assume the following expression instead of Eq. (4):

$$
\varepsilon_{p c \mathrm{II}}^{\prime}=\left[\varepsilon_{p c \mathrm{II} 00}^{\prime} \int_{0}^{\sigma} f_{\mathrm{II}}(x) d x\right] \exp \left(-E_{p c \mathrm{II}} / k T\right),
$$

where $f_{\mathrm{II}}(x)$ denotes a Gaussian distribution function. Figure 12(a) shows the best fitting of Eqs. (3) and (10) to the creep rate vs stress data observed for the type- $H$ specimen. Figure 12(b) shows an example of the Gaussian distribution function $f_{\text {II }}(x)$ found in Fig. 12(a). Values of the stresses at which $f_{\text {II }}(x)$ shows its maxima are $380 \mathrm{MPa}$ at $320 \mathrm{~K}$ and $400 \mathrm{MPa}$ at $295 \mathrm{~K}$, respectively, and are considerably higher than $\sigma_{p c \text { II }}$. This result indicates that the values of the activation enthalpy $E_{p c \text { II }}$ estimated from the application of Eq. (4) should be overestimated because of a temperature change in $f_{\mathrm{II}}(x)$.

It is known that the relationship between the plastic creep rates $\varepsilon_{p c}^{\prime}$, and $\sigma_{a p}$, observed for ductile metal is explained as $\varepsilon_{p c}^{\prime} \propto \sigma_{a p}^{n}$ or $\left(\sigma_{a p}-\sigma_{a p, 0}\right)^{n}$, where $n$ is the stress exponent. For type- $H$ and type- $L$ specimens, the value of $n$ is 1 in region I, as shown in Eq. (3), and is variable with increasing $\sigma_{a p}$ in region II, as shown in Eq. (4). The application of the relationship $\varepsilon_{p c}^{\prime} \propto\left(\sigma_{a p}-\sigma_{p c 2}\right)^{n}$ to the $\varepsilon_{p c}^{\prime}$ data in region II shows that $n$ increases from 1 to 17 with increasing $\sigma_{a p}$ (not shown here), indicating that the assumption for Eq. (10) may be the case. The $\varepsilon_{p c}^{\prime}$ data in region III are limited, and a rough estimation gives $n$ being less than three, indicating that the underlying creep process is not so complicated. In Table 
TABLE I. The creep rate and its stress exponent found for $n$-Au and those reported for $n$-Ni and $n$ - $\mathrm{Cu}$.

\begin{tabular}{|c|c|c|c|c|c|c|c|}
\hline Specimen & $\begin{array}{l}\text { Grain } \\
\text { size } \\
(\mathrm{nm})\end{array}$ & $\begin{array}{c}\text { Preparation } \\
\text { method }\end{array}$ & $\begin{array}{c}\text { Temperature } \\
(\mathrm{K})\end{array}$ & $\begin{array}{c}\text { Applied stress } \\
(\mathrm{MPa})\end{array}$ & $\begin{array}{c}\text { Creep rate, } \\
\varepsilon^{\prime} / \varepsilon_{\text {Ashby }}^{\prime}\end{array}$ & $\begin{array}{c}\text { Stress } \\
\text { exponent } \\
n\end{array}$ & Ref. \\
\hline \multirow[t]{3}{*}{$n-\mathrm{Ni}$} & 6 & Electro deposition & 293 & $500-1000$ & $1.2-1.4$ & 1.18 & 6 \\
\hline & 20 & & 293 & $500-1000^{\mathrm{a}}$ & $0.6-1.0$ & $<2$ & \\
\hline & 40 & & 293 & $400-500^{\mathrm{a}}$ & $0.4-0.5$ & $\sim 2$ & \\
\hline \multirow[t]{2}{*}{$n-\mathrm{Ni}$} & 30 & Electro deposition & 290 & $600-1000$ & 0.3 & 1.1 & 7,8 \\
\hline & & & 373 & $500-700$ & $10^{-4}-10^{-3}$ & 6.5 & \\
\hline$n-\mathrm{Cu}$ & 30 & Electro deposition & $293-323$ & $125-185$ & $10^{-2}-10^{-1}$ & 1 & 4,5 \\
\hline$n-\mathrm{Cu}$ & 10 & GD & 295 & $200-600$ & $10^{-6}$ & $3-8^{\mathrm{b}}$ & 27 \\
\hline \multirow[t]{3}{*}{$n-\mathrm{Au}$} & 20 & GD & $295-320$ & $\begin{array}{l}\text { Region III } \\
\text { near } 360 \mathrm{MPa}(H) \\
\text { near } 500 \mathrm{MPa}(L)\end{array}$ & $0.6-3(H)$ & $<3$ & present \\
\hline & & & & $\begin{array}{l}\text { Region II } \\
150-360 \mathrm{MPa}(H) \\
300-500 \mathrm{MPa}(L)\end{array}$ & $\begin{array}{l}\text { between } \\
\text { regions I } \\
\text { and III }\end{array}$ & $1-17^{b}$ & \\
\hline & & & & $\begin{array}{l}\text { Region I } \\
30-150 \mathrm{MPa}(H) \\
60-300 \mathrm{MPa}(L)\end{array}$ & $\begin{array}{l}10^{-4}(H) \\
10^{-5}(L)\end{array}$ & 1 & \\
\hline
\end{tabular}

${ }^{a}$ Under higher stress, $n$ increased to 5.3, which was claimed to reflect the dislocation (power law) creep in Ref. 6.

${ }^{\mathrm{b}}$ Apparent values which vary with an applied stress. $(H)$ and $(L)$ denote the type- $H$ and type- $L$ specimens.

I, we compiled the ratio of the creep rate observed to $\varepsilon_{\text {Ashby }}^{\prime}$ estimated, assuming the GB diffusion coefficient reported for a coarse grained specimen and the value of $n$. The data reported for $n$-Ni (Refs. 6-8) and those for $n$-Cu (Refs. 4,5, and 27) are also compiled, where $E_{\mathrm{GB}}$ is assumed to be 1.12 $\mathrm{eV}$ reported for $p-\mathrm{N}$ (Ref. 28) and $0.72 \mathrm{eV}$ for $n-\mathrm{Cu},{ }^{4,5}$ respectively. The data at $293 \mathrm{~K}$ for the electrodeposition (ED) $n$-Ni specimens, reported by Wang et al., ${ }^{6}$ suggest that the $\sigma_{a p}$ range between 400 and $1000 \mathrm{MPa}$ for their ED $n-\mathrm{Ni}$ specimens corresponds to region III for the present $n$-Au specimens. Wang et al. ${ }^{6}$ also reported that the value of $n$ increases to 5.3 in the further high $\sigma_{a p}$ range, and claimed that the dislocation power-law creep is responsible for the high value of $n$ found at the high $\sigma_{a p}$ range. The data at 290 $\mathrm{K}$ for the $\mathrm{ED} n-\mathrm{Ni}$ specimens reported by Yin and co-workers ${ }^{7,8}$ are very similar to those reported by Wang et $a l .{ }^{6}$ On the other hand, the creep rate at $373 \mathrm{~K}$ reported in Refs. 7 and 8 appears to show a deviation from their data at $290 \mathrm{~K}$ and the data at $293 \mathrm{~K}$ by Wang et al. ${ }^{6}$ after taking into account a change in temperature, suggesting that the creep mechanism at $373 \mathrm{~K}$ is different from that at room temperature for the ED $n$-Ni specimens. The data between 293 and $323 \mathrm{~K}$ for the ED $n$-Cu specimens reported by Cai et al. ${ }^{5}$ suggest that the $\sigma_{a p}$ range between 125 and $185 \mathrm{MPa}$ for the $\mathrm{ED} n$ - $\mathrm{Cu}$ specimens corresponds to region III for the present $n$-Au specimens. Our preliminary data at $295 \mathrm{~K}$ observed for the GD $n$-Cu specimens ${ }^{27}$ suggest that the $\sigma_{a p}$ range between 200 and $600 \mathrm{MPa}$ for the GD $n-\mathrm{Cu}$ specimens corresponds to region II for the present $n$-Au specimens. From the recent creep data for nanocrystalline face centered cubic metals ( $n$ - $f c c$ metals) compiled in Table I, one can say that the present view of the creep behavior explains the lowtemperature creep behavior of $n-f c c$ metals where the grain growth is not incorporated.
For detailed creep characteristics, one may expect that the creep response of the type- $L n$-Au specimens and that of the type- $H n$-Au specimens become similar to each other in region III, because the GB slip for all the grains may cancel the difference in the GB characteristics, reflecting the deposition condition during the specimen preparation. On the other hand, the $\varepsilon_{p c 3}^{\prime}$ vs $\sigma_{a p}$ data shown in Fig. 4 indicate that the above-mentioned postulation is not the case. It is suggested that the GB slip in region III is governed by the crystallographic relationship between neighboring crystallites, and that the rotation of crystallites is negligible.

\section{CONCLUSIONS}

High-density $n$-Au specimens with a mean grain size of about $20 \mathrm{~nm}$ were prepared by the gas deposition method, where two types of $n$-Au specimens were obtained as a function of a deposition rate, the type- $H$ specimens obtained at the deposition rate above $800 \mathrm{~nm} / \mathrm{s}$ and the type- $L$ specimens obtained at a lower deposition rate. The anelastic and plastic creep responses are very similar between the type- $H$ and type- $L$ specimens qualitatively, but both the anelastic strain and the plastic creep strain are considerably larger for the type- $H$ specimens than for the type- $L$ specimens when they are compared at the same applied stress. The anelastic strain, $\varepsilon_{\text {an GB }}$, associated with a certain anelastic process in the grain-boundary regions, can be observed when the temperature $T$ is higher than the threshold temperature $T_{a n 1}$ of 200 $\mathrm{K}$, and the applied stress $\sigma_{a p}$ is higher than the threshold stress $\sigma_{a n 1}$, of a few MPa. $\varepsilon_{a n, \mathrm{~GB}}$ increases linearly with $\left(T-T_{a n 1}\right)\left(\sigma_{a p}-\sigma_{a n 1}\right)$, and the ratio of $\varepsilon_{a n, \mathrm{~GB}}$ to the elastic strain, $\varepsilon_{e l}$, is as large as 1.1 for the type- $H$ specimens and 0.2 for the type- $L$ specimens at $320 \mathrm{~K}$. The activation en- 
thalpy for the GB anelastic process is as low as $0.2 \mathrm{eV}$. We surmise that the cooperative motions of many atoms in the GB regions are due to $\varepsilon_{a n, \mathrm{~GB}}$, and both the threshold temperature $T_{a n 1}$ and the threshold stress $\sigma_{a n 1}$ show a distribution depending on the number of atoms associated with them.

For the plastic creep response, the creep rate $\varepsilon^{\prime}$ vs $\sigma_{a p}$ data show a letter S-like curve, where we classified the observed creep response into three categories as follows: Region I: the linear creep rate region found for $\sigma_{a p}$ between $\sigma_{p c 1}$ and $\sigma_{p c 2}$. Region II: the transient creep rate region found for $\sigma_{a p}$ between $\sigma_{p c 2}$ and $\sigma_{p c 3}$. Region III: the saturation creep rate region found for $\sigma_{a p}$ between $\sigma_{p c 3}$ and $\sigma_{y}$. $\sigma_{p c 1}, \sigma_{p c 2}$, and $\sigma_{y}$ are about 30,150 , and $360 \mathrm{MPa}$ for the type- $H$ specimens, and about 60, 300, and $500 \mathrm{MPa}$ for the type- $L$ specimens, respectively. $\sigma_{p c 3}$ is slightly lower than $\sigma_{y}$. From STM observations, we surmise that the localized GB slip takes place in region I, and the mean separation between the localized GB slips decreases with increasing $\sigma_{a p}$ in region II and becomes comparable with the mean grain size in region III. The plastic creep in region III may be explained by the Ashby creep. It is suggested that the GB slip in region III is governed by the crystallographic relationship between neighboring crystallites, and a rotation of crystallites is negligible. The present view for the creep behavior explains the low-temperature creep behavior of nanocrystalline $f c c$ metals where the grain growth is not incorporated.

\section{ACKNOWLEDGMENTS}

This work was partly supported by the Center for Tsukuba Advanced Research Alliance (TARA) at the University of Tsukuba, a Grant in Aid for Scientific Research from the Ministry of Education, Science and Culture of Japan, and the High Damping Materials Project of "Research for the Future" of the Japan Society for the Promotion of Science.
*Present address: Takasaki-branch, Advanced Science Research Center, Japan Atomic Energy Research Institute, Watanuki, Takasaki 370-1292, Japan.

†Author to whom correspondence should be addressed. Email address: mizuh@ims.tsukuba.ac.jp

${ }^{1}$ J. P. Poirier, Plasticite a Haute Temperature des Solides Cristallins (Eyrolles, Paris, 1976).

${ }^{2}$ M. F. Ashby and R. A. Verrall, Acta Metall. 21, 149 (1973).

${ }^{3}$ P. G. Sanders, M. Rittner, E. Kiedaish, J. R. Weertman, H. Kung, and Y. C. Lu, Nanostruct. Mater. 9, 433 (1997).

${ }^{4}$ B. Cai, Q. P. Kong, L. Lu, and K. Lu, Scr. Mater. 41, 755 (1999).

${ }^{5}$ B. Cai, Q. P. Kong, L. Lu, and K. Lu, Mater. Sci. Eng., A 286, 188 (2000).

${ }^{6}$ N. Wang, Z. Wang, K. T. Aust, and U. Erb, Mater. Sci. Eng., A 237, 150 (1997).

${ }^{7}$ W. M. Yin, S. H. Whang, R. Mirshams, and C. H. Xiao, Mater. Sci. Eng., A 301, 18 (2001).

${ }^{8}$ W. M. Yin and S. H. Whang, Scr. Mater. 44, 569 (2001).

${ }^{9}$ G. W. Nieman, J. R. Weertman, and R. W. Siegel, Scr. Metall. 23, 2013 (1989).

${ }^{10}$ D. Korn, A. Morsch, R. Birringer, W. Arnold, and H. Gleiter, J. Phys. (Paris), Colloq. 49, C5-769 (1988).

${ }^{11}$ M. Weller, J. Diehl, and H.-E. Schaefer, Philos. Mag. A 63, 527 (1991)

${ }^{12}$ P. G. Sanders, A. B. Witney, J. R. Weertman, R. Z. Valiev, and R. W. Siegel, Mater. Sci. Eng., A 204, 7 (1995).

${ }^{13}$ P. G. Sanders, J. A. Eastman, and J. R. Weertman, Acta Mater. 45, 4019 (1997).

${ }^{14}$ P. G. Sanders, C. J. Youngdahl, and J. R. Weertman, Mater. Sci. Eng., A 234-236, 77 (1997).
${ }^{15}$ X. Y. Qin, X. R. Zhang, G. S. Cheng, and L. D. Zhang, Nanostruct. Mater. 4, 661 (1998).

${ }^{16}$ S. Sakai, H. Tanimoto, and H. Mizubayashi, Acta Mater. 47, 211 (1999).

${ }^{17}$ S. Sakai, H. Tanimoto, K. Otsuka, T. Yamada, Y. Koda, E. Kita, and H. Mizubayashi, Scr. Mater. 45, 1313 (2001).

${ }^{18}$ H. Tanimoto, S. Sakai, K. Otsuka, E. Kika, and H. Mizubayashi (unpublished).

${ }^{19}$ H. Tanimoto, S. Sakai, and H. Mizubayashi, Nanostruct. Mater. 12, 751 (1999).

${ }^{20}$ H. Tanimoto, S. Sakai, and H. Mizubayashi, Trans. Mater. Res. Soc. Jpn. 24, 117 (1999).

${ }^{21}$ W. B. Li, J. L. Henshall, R. M. Hooper, and K. E. Eastering, Acta Metall. Mater. 39, 3099 (1991).

${ }^{22}$ E. A. Brandes, Smithells Metals Reference Book, 6th ed. (Butterworths, London, 1983).

${ }^{23}$ E. Bonetti, E. G. Campari, L. Del Bianco, L. Pasquini, and E. Sampaolesi, Nanostruct. Mater. 11, 709 (1999).

${ }^{24}$ P. Keblinski, D. Wolf, S. R. Phillpot, and H. Gleiter, Philos. Mag. A 79, 2735 (1999).

${ }^{25}$ B. Shönfelder, D. Wolf, S. R. Phillpot, and M. Furtkamp, Interface Sci. 5, 245 (1997).

${ }^{26}$ B. Shönfelder, P. Keblinski, D. Wolf, and S. R. Phillpot, Mater. Sci. Forum 294-296, 9 (1999).

${ }^{27}$ H. Tanimoto, N. Yagi, S. Sakai, E. Kita, and H. Mizubayashi, Mater. Jpn. 41, 410 (2002).

${ }^{28}$ I. Kaur, W. Gust, and L. Kizma, Handbook of Grain and Interphase Boundary Diffusion Data (Ziegler, Stuttgart, 1989), Vol. 1. 